\title{
A Child with Intra-orbital Oculomotor Nerve Schwannoma without Neurofibromatosis
}

\author{
Muhammad Shahzad Shamim, Muhammad Ehsan Bari, Khalid Nasim Chisti, Asad Abbas
}

Can. J. Neurol. Sci. 2008; 35: 528-530

Orbital tumors constitute a heterogeneous array of lesions and pose numerous challenges in terms of diagnosis, imaging and treatment. ${ }^{1,2}$ The bony orbit contains an extremely complicated anatomical architecture composed of II, III, IV, VI cranial nerves, vessels, extra ocular muscles and the globe. Schwannoma of cranial nerves represent less than $8 \%$ of all intracranial neoplasms usually arising from the vestibulocochlear nerve. These tumors are commonly seen in association with Neurofibromatosis and constitute approximately $1 \%$ of all orbital tumors. ${ }^{3}$ To date there are only 38 reported cases of orbital tumors arising from the oculomotor nerve, and only four arising within the orbit. ${ }^{4,5}$

\section{CASE RePORT}

An 11-year-old girl presented to the clinic with a three year history of slowly progressive, painless protrusion of the left eye, drooping of the left eyelid, gradual worsening of vision and concomitant limitation of eye movement. On examination she was blind in the left eye with complete paralysis of extra ocular movement. A mass was visible at outer sclera along with exophthalmos, infro nasal deviation of the eyeball and severe esotropia. (Figures 1a, 1b). The right eye had 20/20 vision and normal movements in all directions. Apart from the positive findings in her left eye, patient had normal examination of the rest of her cranial nerves (including hearing). She had normal examination of her higher mental functions, with no nystagmus or Romberg sign; no hyperreflexia or other corticospinal tract sign, and no cerebellar deficits. The rest of the general physical and system examination was also normal with no signs of Neurofibromatosis. She was born of consanguineous marriage and had two older brothers. All her family members were healthy.

Magnetic resonance imaging (MRI) revealed a well defined large intra-conal mass in the left orbit, extending up to the optic canal but not into it. (Figures $2 \mathrm{a}, 2 \mathrm{~b}, 2 \mathrm{c}$ ). This mass was isointense to hypo-intense on T1 weighted image and hyper-intense on T2. There was marked nodular enhancement with some non enhancing hyper-intense areas within it. The mass was causing mild proptosis and circumferentially surrounding the optic nerve, which did not show any expansion. The left extra ocular muscles were displaced peripherally due to this mass. There were no abnormalities of the optic chiasm and tracts. With a provisional diagnosis of an intraconal space occupying lesion, excisional biopsy was planned.

Exposure was obtained through a slightly curved skin incision made below the left eyebrow, orbital fascia was opened and the tumor was found almost reaching the surface. Using standard microsurgical technique, the tumor was dissected free from the globe to which it was attached by flimsy fibrous tissue, divided at the optic foramen, and removed. No intra orbital structures were damaged in the process. No direct association with any of the cranial nerves could be established. Histopathology showed spindle cells arranged in fascicles with intervening loose areas and thick walled vessels. Immunohistochemical stain showed diffuse positivity of spindle cells for S100. The patient's post operative course was uneventful with moderate residual ptosis, mild to moderate esotropia and paresis of superior and lateral rectus muscles but definite cosmetic improvement (Figure 3). There was no recovery of vision as expected and explained to the patient pre-operatively. Follow up to one year was unremarkable.

\section{Discussion}

Orbital tumors are rare clinical entities that can be classified based on their origin, into primary lesions which originate from the orbit itself; secondary lesions, that extend to the orbit from neighboring structures; and metastatic tumors. They may also be classified anatomically into intraconal and extraconal lesions, depending upon their relationship with the muscle cone formed by the extraoccular muscles and intermuscular septae.

The two most common primary peripheral nerve sheath neoplasms are neurofibromas and schwannomas. In sporadic cases, schwannomas usually arise from sensory nerves or from the nerve sheath of the eighth cranial nerve and only rarely are found arising in the orbit. The MRI with gadolinium-based contrast enhancement is the imaging modality of choice for such tumors. Computed tomography (CT) is also helpful as it is more sensitive in depicting adjacent bone destruction. Excisional biopsy is the only definitive means of diagnosis. Microscopically, these tumors show areas of densely packed spindle cells termed Antoni A tissue, intermixed with loose, myxoid regions termed Antoni B tissue. Surgery remains the mainstay of therapy for the majority of symptomatic orbital tumors, including orbital schwannomas. The lesion is solid and encapsulated by the perineurium of the nerve from which it

From the Department of Neurosurgery, Aga Khan University Hospital (MSS, MEB, KNC), Karachi, Pakistan; Department of Ophthalmology, Southeast Texas Medical Associates (AA), Beaumont, TX, USA.

Received August 20, 2007. Final Revisions Submitted April 21, 2008.

Correspondence to: M Ehsan Bari, Department of Neurosurgery, Aga Khan University, Stadium Road, P.O. Box 3500, Karachi-74800, Pakistan. 


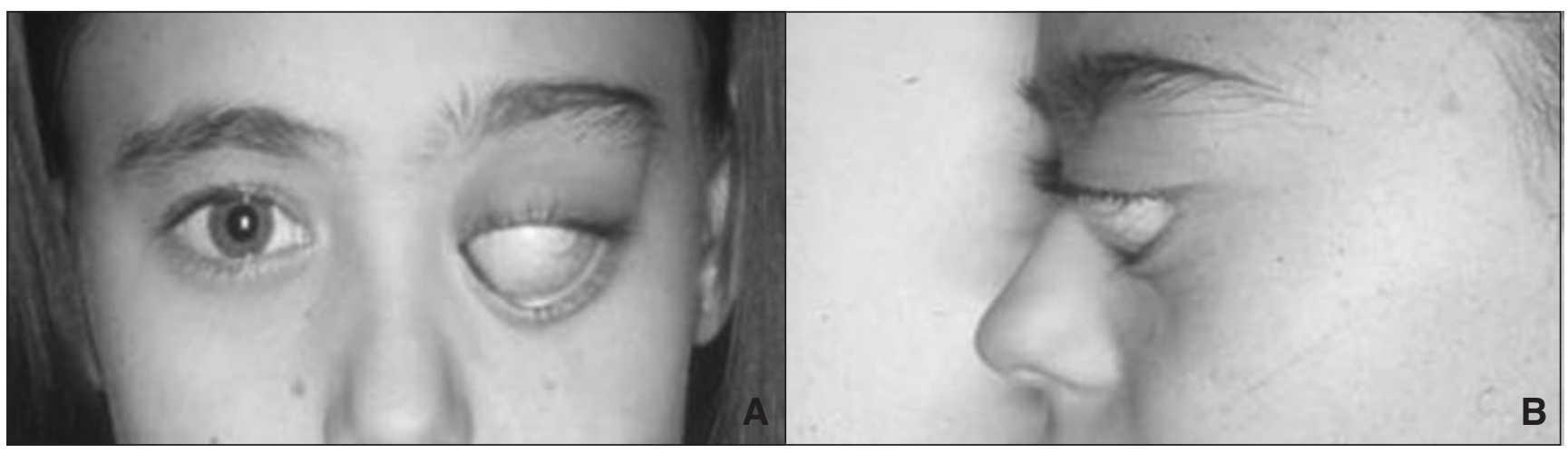

Figure 1A \& B. Preoperative pictures of the girl showing proptosis and deviation of the left eye.

arises and is thus relatively amenable to total resection. Surgery not only provides a diagnosis, which is essential, but is also warranted for prevention of disease progression as well as providing relief from the disfiguring proptosis. Functional recovery may not be possible in long standing tumors with loss of vision.

Our patient had no family history, nor any other signs to suggest the presence of neurofibromatosis. She was thus diagnosed to have the rare, but not unknown, occurrence of sporadic form of schwannoma, developing as a primary intraconal lesion, most probably arising from the oculomotor nerve as she developed ptosis prior to her other symptoms. Although the oculomotor nerve could not be identified as the source of this tumor, it is not unusual to find that the anatomical relationships between the tumor and its hosting nerve are not well defined, especially in cases of large tumors. In a series of seven cases of primary orbital schwannoma reported by Rootman et al, the nerve of origin of only four of the tumors could be identified. ${ }^{6}$ It has been proposed that third nerve palsy as the initial symptom of such a tumor may be pathognomonic for oculomotor nerve sheath tumors, as was in our patient. ${ }^{7}$ Two clues leading to the diagnosis are an oculomotor paresis and tumor location along the course of the oculomotor nerve, especially in its cisternal portion. Henderson et al in a review of 1376 orbital lesions reported that only $3.9 \%$ were nerve sheath tumors, with only 15 (approximately 1\%) schwannomas. ${ }^{2}$ They present either as a painless progressively increasing mass, with proptosis, or symptoms attributed to compression neuropathy. Mariniello and colleagues claimed, in 1999, that there were less than 25 reported cases of tumors arising from the oculomotor nerve not associated with Neurofibromatosis. ${ }^{5}$ To the best of our knowledge, to date only 38 cases of oculomotor nerve schwannomas have been reported in the literature, including 15 male and 23 female patients with an age range from 8 to 74 years..$^{3,5-14}$ Preoperative oculomotor dysfunction was manifested in 30 cases out of 38 , and also in our patient. The tumor was located in the orbit in just

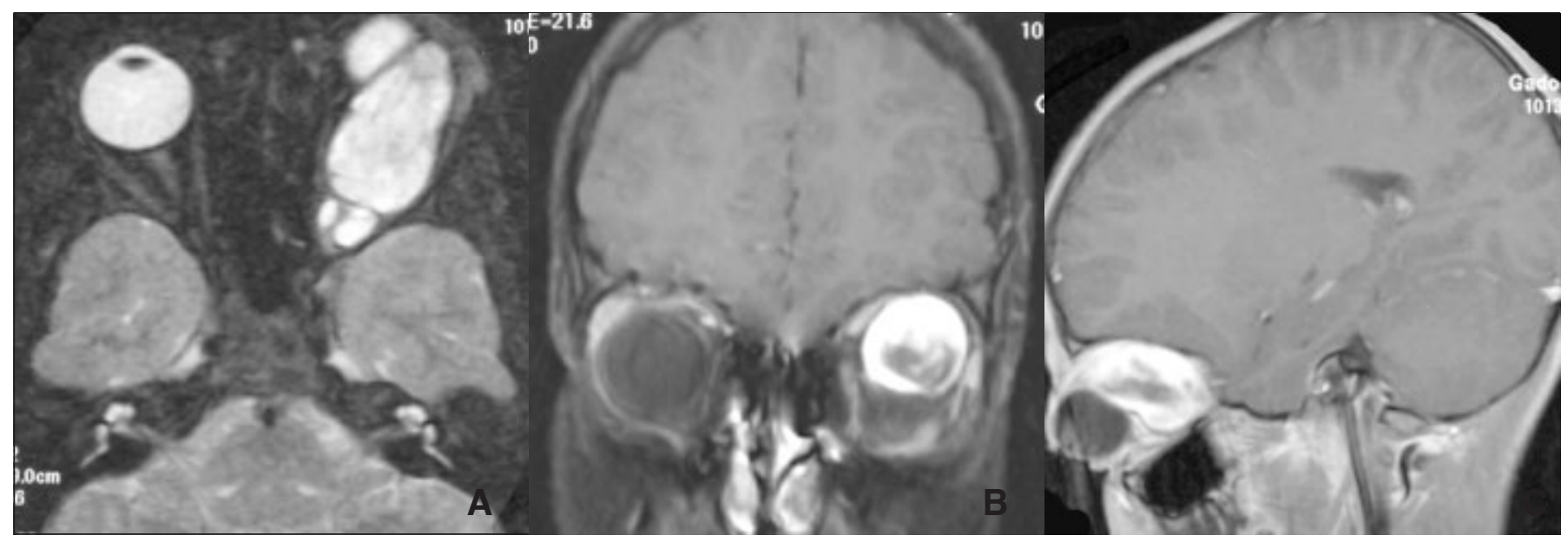

Figure $2 A, B \& C . M R I T 1$ weighted pre and post contrast (axial pre contrast, sagittal and coronal post contrast) images showing orbital tumor compressing the globe causing optic nerve compression and proptosis. 


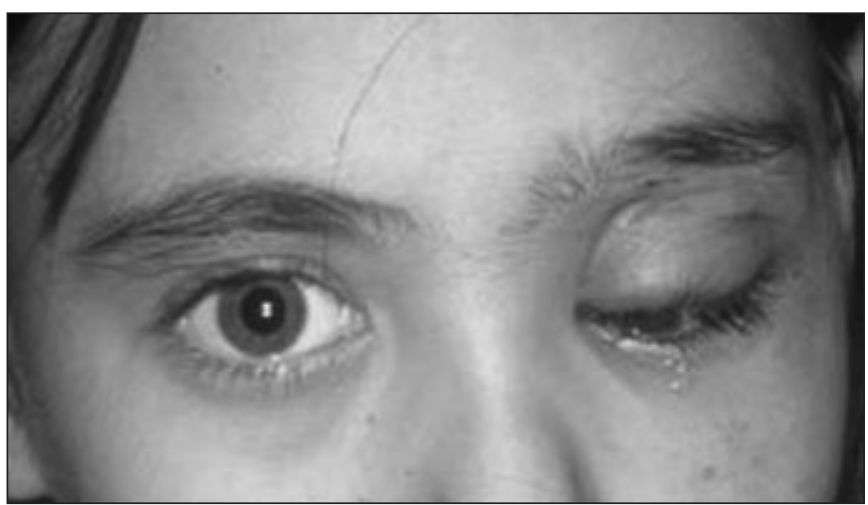

Figure 3: Postoperative improvement in proptosis with moderate residual ptosis.

four cases (solitary orbital type), in the subarachnoid space in 17 cases (cisternal type), in the cavernous sinus in 12 cases (cavernous type), extending from the cavernous sinus to the cistern in five cases (cisterno-cavernous type) and extending from cavernous sinus to the orbit in one case. , $^{3,-14}$ Our case is unusual due to a number of reasons. The age of our patient is of interest as most of these cases have been reported in middle aged patients with the youngest reported patient being only eight years old. $2,6,13,14$ Also this, to the best of our knowledge, is only the fifth case report of intra-orbital oculomotor nerve schwannoma. ${ }^{3,5-14}$ Schwannomas of the oculomotor nerve have also been reported to develop in unusual places, corresponding to the path of the nerve, such as inside the para-sellar region or from the segment of the nerve in the inter-peduncular cistern. Occasionally a large lesion may affect the nerve more extensively, from the brainstem to the cavernous sinus, occupying both the middle and posterior cranial fossae..$^{9,12}$

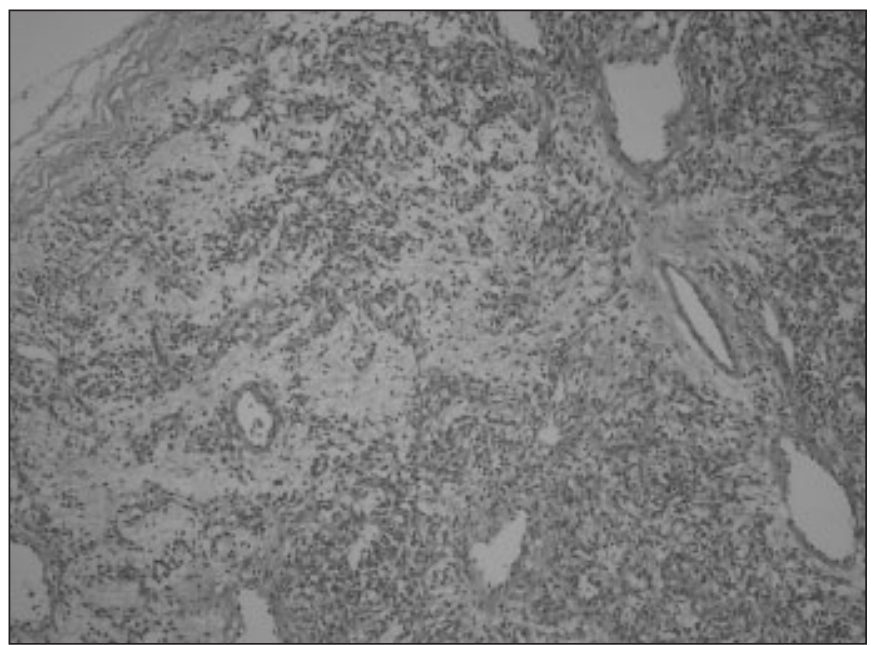

Figure 4: Haematoxylin and eosin stain shows spindle cells arranged in short fascicles with intervening loose areas along with thick walled vessels.

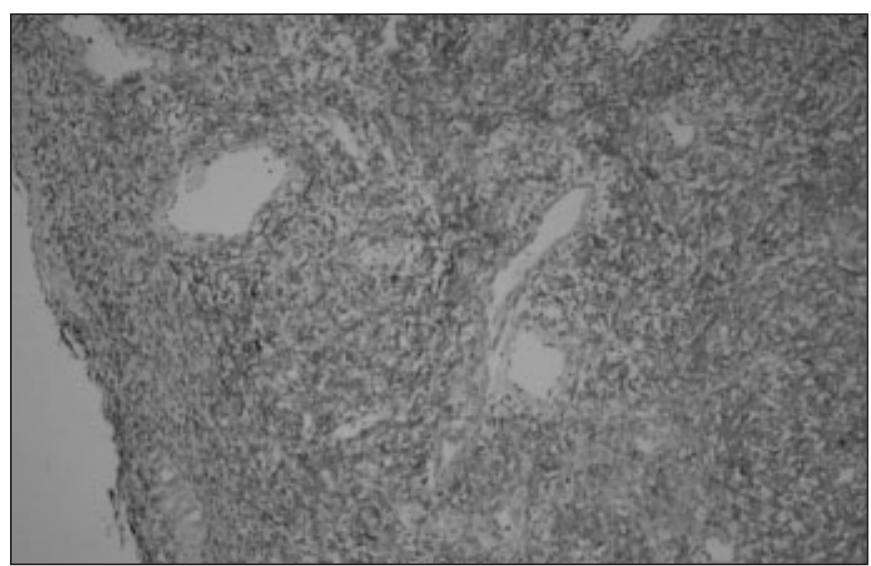

Figure 5: Immunohistochemical stain shows diffuse positivity of spindle cells for $\mathrm{S100.}$

\section{ACKNOWLEDGEMENT}

The authors thank Professor Terrence Myles (University of Calgary) for help and support during the preparation of this manuscript and Professor Sheema Hassan (Department of Pathology, Aga Khan University Hospital) for her help in preparing and labeling the histological slides.

\section{REFERENCES}

1. Barat JL, Marchal JC, Bracard S, Auque J, Martin-Beuzart S, Hepner H. [Neurinoma of the oculomotor nerves. Apropos of 2 cases]. Neurochirurgie. 1992;38:183-7.

2. Henderson JW. Orbital tumors. 3rd ed. New York: Raven Press; 1994.

3. Konrad EA, Thiel HJ. Schwannoma of the orbit. Ophthalmologica. 1984;188:118-27.

4. Kenji O, Toshihiro T, Takeo G, Kenichi I. Schwannoma of the oculomotor nerve. Neurol India. 2006;54(4):437-9.

5. Mariniello G, Horvat A, Dolenc V. En bloc resection of an intracavernous oculomotor nerve schwannoma and grafting of the oculomotor nerve with sural nerve. J Neurosurg. 1999:91:1045-9.

6. Rootman J, Goldberg C, Robertson W. Primary orbital schwannomas. Br J Opthalmol. 1982; 66:194-204.

7. Okamoto S, Handa H, Yamashita J. Neurinoma of the oculomotor nerve. Surg Neurol. 1985;24:275-8.

8. Asaoka K, Sawamura Y, Murai H, Satoh M. Schwannoma of the oculomotor nerve: a case report with consideration of the surgical treatment. Neurosurgery. 1999;45:630-4

9. Hiscott P, Symon L. An unusual presentation of neurofibroma of the oculomotor nerve. Case report. J Neurosurg. 1982;56:854-6.

10. Kachhara R, Nair S, Radhakrishnan VV. Oculomotor nerve neurinoma: report of two cases. Acta Neurochir. 1998; 140: 1147-51.

11. Katoh M, Kawamoto T, Ohnishi K, Sawamura Y, Abe H. Asymptomatic schwannoma of the oculomotor nerve: case report. J Clin Neurosci. 2000;7:458-60.

12. Mehta VS, Singh RVP, Misra NK, Choudhary C. Schwannoma of the occulomotor nerve. Br J Neurosurg. 1990;4:69-72.

13. Murakami T, Funatsuka M, Komine M, Hirayama Y, Suzuki H, Shishikura K, et al. Oculomotor nerve schwannoma mimicking ophthalmoplegic migraine. Neuropediatrics. 2005;36(6):395-8.

14. Netuka D, Benes V. Oculomotor nerve schwannoma. Br J Neurosurg. 2003;17(2):168-73. 